\title{
Advancements in Epigenetic Research and Its Role in Cancer Therapy
}

Anita Liu (McGill University)

News Reporter - HSI 2OIO-2OII

In the past, cancer was predominantly viewed as a genetic disease, thus implying our biology is our destiny. In recent years, the scientific community has slowly recognized that although our DNA will not change throughout our lifetime, non-genetic factors, such as social environment, can influence the way our genes are expressed (e.g. by altering DNA methylation). These changes can be quite robust, often resulting in phenotypic changes. This phenomenon coined the term "epigenetic", which is defined as a change in gene function that does not involve changes in DNA sequence [1]. Following this discovery, a much more complex picture of cancer was painted and one Canadian scientist, Dr. Moshe Syzf, has greatly contributed to what we've learned so far.

Dr. Moshe Szyf is a James McGill professor in the Department of Pharmacology \& Therapeutics at McGill University, and a pioneer in the field of epigenetics. Dr. Szyf conducts interdisciplinary research and investigates DNA methylation patterns in diabetes, epilepsy, suicide, varying socioeconomic classes and cancer. Dr. Szyf was named Scientist of the Year in 2009 by Radio-Canada alongside his research collaborators, Drs. Michael Meaney (McGill) and Gustavo Turecki (McGill), for their work on the epigenetic effects of child abuse on the human brain [2]. Health Science Inquiry was fortunate to have the opportunity to conduct an interview with Dr. Szyf, where he described how DNA methylation relates to cancer, what his lab is currently working on with international collaborators, and how cancer research has progressed throughout the years.

\begin{abstract}
What is DNA methylation and how does it relate to cancer? DNA methylation is kind of the punctuation mark of the genome, and these punctuations vary substantially from tissue to tissue. In cancer, these normal patterning of punctuations is altered. We are currently working on mapping methylation [patterns] of different cancers to see if we can get a signature and subsequently try to differentiate cancerous vs. normal cells. We don't think it's one specific mark, but rather a signature. The genome has a signature, which provides us an identity: it's almost like an iris reader in an airport, and if we can define this cancer identity we can compare it to healthy cells [to use it as a diagnostic tool].
\end{abstract}

\section{What is your lab currently working on?}

The project that we are working on right now is liver cancer with collaborators in China. Liver cancer is very interesting because early detection has almost a $100 \%$ recovery in contrast to late detection, which has a poor prognosis. Most people are diagnosed very late and thus, death is almost $100 \%$. The challenge is that not all inflamed livers develop into cancer, so how do you detect those that are cancerous from those that aren't?

\section{Is there a particular reason why you are focusing on liver cancer?}

Not in particular. Liver cancer is very common in certain places - it's a great model to prove the principle [i.e. DNA methylation patterns can be used as a cancer detection tool], and if it works we can go to breast and prostate cancer. You want to aim for a cancer where there is no drug and if it works, you can apply it to other cancers. Also,

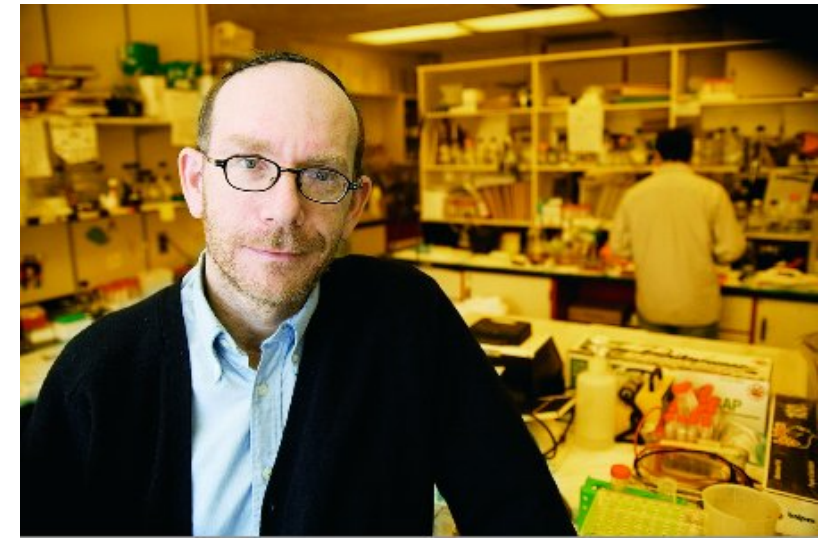

opportunities in China are amazing; the way the medical system is organized is that it's very centralized so it's easier to recruit patients and it is cheaper to conduct clinical trials there.

\section{How long does it take for a drug to become available?}

It depends on money. Clinical trials are expensive and cost millions of dollars [in China]... in the west, it'll cost hundreds of millions of dollars. There isn't an agency that funds this, especially new clinical trials - only private investors or pharmaceutical companies will. The economic climate in China is very risk adverse and they will only want to invest in clinical trials in things that they know for sure will work, but you can't guarantee that. It's unfortunate that this is the major roadblock all over the world; you need to convince somebody to throw us 6 million dollars, but you can't guarantee them it'll work. 
How has research in cancer progressed over the years?

The classic definition of cancer as a molecular disease, which was very dominant in the 80 s and 90s [and subsequently led to the discovery of things like oncogenes], [stems the concept] that cancer is a systemic disease. However, the cell is just a phenotype rather than the cause of the phenotype. [It's been shown now that] social stress can activate pathways that will change an expression [pattern to one] that can cause cancer. Now we understand that it's not just a network of a cell, organ, or body - it's a network of an environment, and that environment is the combination of the physical and social environment. There was also a whole issue of causality and doing simple experiments where you knock out one gene to see one phenotype and it was very naïve - now we realize that the same protein can be cancer promoting or cancer suppressing. So, there's a movement from a simplistic linear thinking to a circular thinking.

\section{What are the challenges for cancer research today?}

Now the challenge is to figure out these [networks of] circuits and how we design therapeutics that take into account these circuits. In the old days, we wanted to use a specific drug, but now we understand that a specific drug is a very bad idea because it only knocks out one element of a circuit, which probably won't do much.
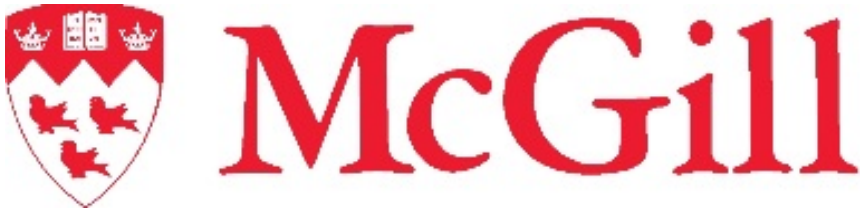

Department of

PHARMACOLOGY \& THERAPEUTICS age 8000

\section{And finally, what do you do for fun and what is the 'fun' aspect of research?}

I have zero free time. I do a lot of traveling, but not for fun (for work). So, I know many taxis and hotels and I usually don't have time to see anything. But, the fun part is meeting new people [because I have many international collaborators in Asia, Europe and America] and seeing the different ways they do science. As much as science is supposed to be objective, it reflects their culture - and I think it's great! It would be very sad to see science only being done one way. So, I think it's not outside of my work, but it's the fun part of my work.

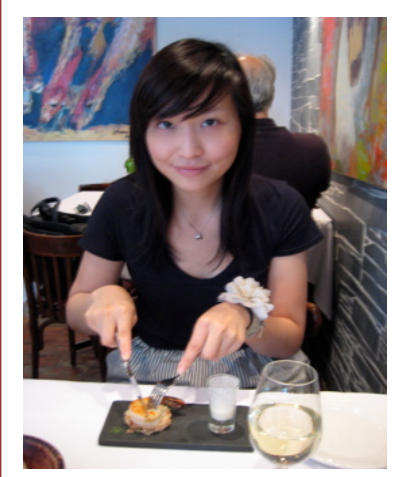

News Reporter Profile

Anita Liu is currently pursuing a MSc in Neurology \& Neurosurgery at McGill University. Aside from neurology research, her interests include disseminating accurate and relevant health information to the public, promoting science and health education in Aboriginal and immigrant communities, as well as learning about new cultures. 